\title{
APPLICATION OF THE CONJUGATE GRADIENT METHOD TO A SELF-MATCHING NETWORK FOR INDUSTRIAL MICROWAVE HEATING ANTENNAS
}

\author{
Jordi J. Mallorquí (*), Albert Aguasca, Angel Cardama, Romà Pagès, Josep \\ Haro \\ Electromagnetic Engineering and Photonics Group \\ Dept. of Signal Theory and Communications, Universitat Politècnica de Catalunya \\ D3-Campus Nord-UPC, C/ Jordi Girona 1-3, 08034 Barcelona (Spain) \\ E-Mail: mallorqu@voltor.upc.es; Tel: 34-93 40172 29; Fax: 34-93 4017232
}

\section{Introduction}

One of the main problems regarding the efficiency in a heating industrial system using high power microwaves is related with the uncontrolled changes on the load. These changes can be caused both by the different kinds of materials to be processed in the same oven and by the temperature rise which modifies the material's physical properties, particularly its dielectric permittivity. Usually, the microwave generator is matched to the antenna feeding the oven by means of a waveguide matching system, typically a two, three or four capacitive-screw tuner empirically adjusted for a given load [1]. Once the heating process is started, the temperature dependence of the complex permittivity usually changes the impedance seen by the generator, worsening the mismatch factor and reducing the efficiency of the heating process. A similar happens when the load is inhomogeneous or discontinuous; it is not feasible to manually re-adjust the matching network for every different sample processed. Until now the solution has been simple but highly inefficient: to increase the incident power in order to overcome the mismatching effects.

The Magic $\mathrm{T}$ as a matching network

The magic $T$ is the waveguide implementation of a $180^{\circ}$ hybrid junction, a fourport network which has a $180^{\circ}$ phase shift between the two output ports [2] Considering an ideal magic $T$ with sliding shorts at the $E$ and $H$ planes, the input reflection coefficient, $\Gamma_{i n}$, can be expressed as a function of the load reflection coefficient, $\Gamma_{L}$, and the electrical phases corresponding to the positions of the two sliding shorts, $\Gamma_{E}=e^{j \Phi_{E}}$ and $\Gamma_{H}=e^{j \Phi_{H}}$.

$$
\Gamma_{i n}=\frac{1}{2}\left(\frac{2\left(\Gamma_{E}+\Gamma_{H}\right)-4 \Gamma_{L} \Gamma_{E} \Gamma_{H}}{2-\left(\Gamma_{E}+\Gamma_{H}\right) \Gamma_{L}}\right)
$$


Several stub positions that match a given lossy load can be found, the number depending on their electrical length. Despite this simple relationship between $\Gamma_{L}$ and the stub positions, it is not easy to implement it in an industrial microwave oven because it requires the knowledge of $\Gamma_{L}$ in both magnitude and phase. $A$ high power vector network analyser should be implemented and included in the oven. In addition, the working frequency of an industrial magnetron depends on the load, the so-called frequency pulling [3], and even when the load does not change the magnetron's frequency stability is poor. With highly reactive loads the bandwidth of the matching network is narrow, so small frequency deviations could cause a large mismatch, even with a constant load. Therefore, a dynamic matching network able to react in front of both load and frequency changes is needed. The last practical aspect, but not the least important, is that even when the correct position of the stubs could be computed, during its positioning a temporal worsening of the mismatch losses will be produced

\section{The Algorithm}

The algorithm is based on the well-known Conjugate Gradient Method, CGM, used to iteratively minimize functions which depend on one or more independent variables [4]. At each step the algorithm performs a linear minimization of the function along a direction computed by using the local gradient and all the previous directions traversed. For quadratic functions, the procedure generates a set of mutually conjugated directions that correctly weighted by the algorithm reach to the function minima. Unfortunately, in the real world functions representing real processes usually are not quadratic. Despite this in most cases the CGM can be still used because functions usually present a quasi-quadratic behaviour near the minima. In such cases, the number of iterations required to get the minima will be larger than the number of independent variables and will depend on both the kind of function and the starting point. When the function has different local minima the CGM does not warrant the global minimum.

The benefit of using CGM is that only requires function evaluations, and not the algebraic knowledge of the function to be minimized. The required gradient evaluations can be computed by using a typical finite difference scheme. In this application the initial problem has been reduced to the minimization of the unknown near quadratic function, $\left|\Gamma_{\text {in }}\right|=f\left(\Gamma_{L}, \Gamma_{E}, \Gamma_{H}\right)$, by means of successive function evaluations in a conjugate gradient scheme.

\section{The Hardware}

Two short circuits have been implemented in a circular waveguide, whose dimensions are near the cut-off frequency of the $\mathrm{TE}_{11}$ mode in order to reduce its wavelength to the minimum. The short plungers have been designed by using a folded half-wavelength capacitance coupling [5]. The measurement of the reflection coefficient inside the rectangular waveguide has been 
implemented with a double directional coupler using a hybrid technology (waveguide-microstrip) which minimizes its volume [6]. Two power detectors at the coupled branches provide a voltage proportional to the power carried by the incident and reflected waves. Finally, one microcontroller, a Microchip $\mathrm{PIC16C74,} \mathrm{runs} \mathrm{the} \mathrm{CGM} \mathrm{with} \mathrm{the} \mathrm{measurements} \mathrm{and} \mathrm{controls} \mathrm{the} \mathrm{stubs}$ positions by means of a couple of step-by-step motors. The experimental prototype can be seen in the Fig.1.

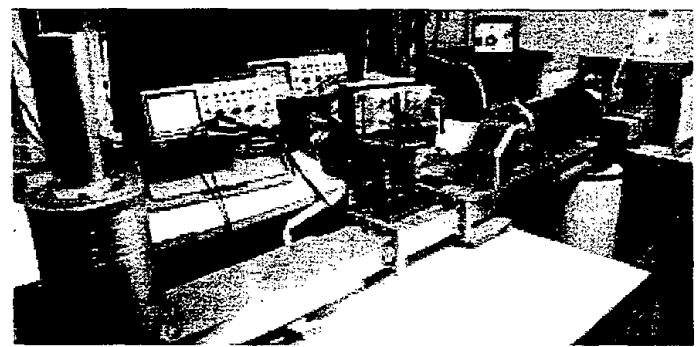

Fig. 1 Experimental prototype. From background to forefront, the magnetron, the magic $T$ with the two cylindrical stubs, the box with the microcontrolier and auxiliary electronics, and the cavity.

\section{Results}

The system has shown its capability to automatically match changing loads inside an industrial microwave oven. Among the different tests carried out, the drying of a wet sponge is presented in order to show the typical behaviour of a changing load inside a small cavity. Fig.2 (a) shows the temporal evolution of the input reflection coefficient depending on the matching network used. When a manual three-screw tuner is used it is difficult to achieve more than $10 \mathrm{~dB}$ and, once the water starts to evaporate, the mismatching increases presenting an oscillatory behaviour. If the automatic matching network is placed between the magnetron and the load, the CGM scheme is able to maintain an average return loss better than $15 \mathrm{~dB}$. The benefits of the automatic matching network are evident at the end of the drying process, when the reduced levels of water inside the sponge make the load more reactive. Fig. 2 (b) presents the energetic efficiency for both cases in terms of the power transfer percentage. While the automatic network assures more than a $95 \%$ of efficiency for most of the drying process, the manual one only stays around $75 \%$, falling to $25 \%$ at the end of the process.

\section{Conclusions}

An automatic matching network for microwave industrial applications based on a conjugate gradient scheme has been presented. The combining of a waveguide $180^{\circ}$ hybrid junction with two sliding short circuits controlled by a 
microcontroller has shown its potential capability to increase the efficiency of industrial processes involving unknown or changing highly reactive loads in microwave ovens.
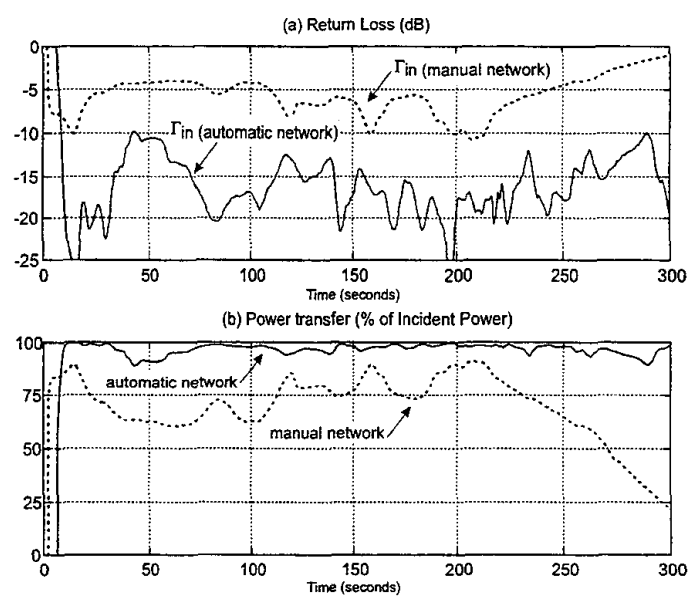

Fig. 2(a) Comparison between the temporal behaviour of the return loss when a changing load is manually adjusted with a three screw tuner,

$\Gamma_{i n}$ (manual network), and when the automatic magic $\mathrm{T}$ is used,

$\Gamma_{\text {in }}$ (automatic network). (b) The transferred power from magnetron to load is presented for both cases.

\section{Acknowledgements}

This work has been sponsored by the company CMM-D'OR and the Generalitat de Catalunya under grant CIRIT-CIDEM.

\section{References}

[1] G. Roussy, J. A. Pierce, "Foundations and Industrial Applications of Microwave and Radio Frequency Fields", John Wiley \& Sons, 1995.

[2] D. M. Pozar, "Microwave Engineering", Addison-Wesley Publishing Co., 1993.

[3] A. S. Gilmour Jr.. "Microwave Tubes", Artech House, 1986.

[4] W. H. Press, B. P. Flannery, "Numerical Recipes in C: The Art of Scientific Computing", Cambridge University Press, 1989.

[5] G. L. Ragan, "Microwave Transmission Circuits", Dover Publications, Inc. 1968.

[6] M. Caron, C. Akyel, F. M. Ghannouchi, "A versatile Easy to do Six-Port Based

High Power Reflectometer", Journal of Microwave Power and Electromagnetic

Energy, International Microwave Power Institute, pp. 231-239, Vol. 30, No. 4, 1995 